\title{
Securing Multi-channel Selection using Distributed Trust in Cognitive Radio Ad hoc Networks ${ }^{\text {th }}$
}

\author{
Jingyu Feng*1,1, Xu Du ${ }^{1}$, Guanghua Zhang ${ }^{1}$, Wei Shi ${ }^{1}$ \\ ${ }^{a}$ Department of Communication Engineering, Xi'an University of Posts and \\ Telecommunications, Xi'an 710121, China \\ ${ }^{b}$ State Key Laboratory of Information Security (Institute of Information Engineering), \\ Chinese Academy of Sciences, Beijing 100093 \\ ${ }^{c}$ College of Information Science and Engineering, Hebei University of Science and \\ Technology, Shijiazhuang 050000, China
}

\begin{abstract}
Cognitive radio ad hoc networks (CRAHNs) which are independent with infrastructure and fixed spectrum allocation policy, have been developed rapidly in the environment of scarce spectrum resources. However, the nature of multi-channel makes CRAHNs offers opportunities for malicious SUs. Cooperative spectrum sensing (CSS) is the key function of CRAHNs to identify the idle spectrum as the available channel by aggregating sensing data. Malicious SUs can launch spectrum sensing data falsification (SSDF) attack against CSS, and thus causing the disturbance of finding multi-channel. Recently, a lot of efforts with trust schemes have been paid to combating SSDF attack in central cognitive radio environments, but little attention to the distributed environments. In this paper, we propose a distributed trust evaluation (DTE) scheme from the perspective of direct experience and neighbor help to counter SSDF attack, which can be implemented at each SU in CRAHNs rather than a central authority. To secure multi-channel selection fully, it is very important to avoid the conflict among

\footnotetext{
This research was supported in part by the National Science Foundation of China (61301091), the Open Foundation of State Key Laboratory of Information Security (2015MS-14), the Industrial Science and Technology Project of Shaanxi Province (2016GY-113), the Science Foundation of Shaanxi Provincial Education Office (15JK1649), the University Scientific Research Foundation of Hebei Province of China (YQ2014036), the China Postdoctoral Science Foundation (2015M582622), the New Star Team of X'an University of Posts \& Telecommunications.

${ }^{*}$ Corresponding author: Jingyu Feng, E-mail: fjyu1984@163.com
} 
SUs for competing idle channels. We also design a distributed trust implementation protocol to manage CSS with the DTE scheme in a distributed manner. Simulation results show that our scheme can successfully suppress SSDF attack at a low cost, and thus ensure the security of multi-channel selection in CRAHNs.

Keywords: Cognitive radio, ad hoc, cooperative spectrum sensing, multi-channel, trust.

\section{Introduction}

With the rapid development of wireless communication and the huge demand of the capacity for wireless applications, the wireless frequency spectrum has become increasingly scarce. At the same time, a large portion of the assigned

5 spectrum bands, such as in the 400-700 MHz range, that are used sporadically or under-utilized for transmission [1]. To solve the contradiction between the spectrum scarcity and low spectrum utilization, cognitive radio has been considered as a useful technology. Currently, cognitive radio technology is introduced in ad hoc networks, and thus forming cognitive radio ad hoc networks (CRAHNs) which refer to the distributed networks where unlicensed users (or called secondary users-SUs) can share the idle spectrum with licensed users (or called primary users-PUs) if they do not cause any interference to PUs [2]. CRAHNs are gaining importance with the increasing number of potential applications, such as military battlefield communications, disaster relief, and autonomous vehicular communications [3].

Unlike traditional ad hoc networks, multi-channel is the main feature of CRAHNs, in which each SU needs to sense the idle spectrum of PUs while forwarding data packets. Cooperative spectrum sensing (CSS) is the key function of CRAHNs to find the available channel by exploiting spatial diversity via the observations of spatially located SUs. In a CSS architecture, all the participating SUs send their observations regarding the presence or absence of PU signals to the source SU, which makes the final decision about whether a PU spectrum 
is idle or not. However, aggregating data in CSS makes CRAHNs offer opportunities for malicious SUs to launch SSDF [4] attack by sending false spectrum sensing data against multi-channel routing. For convenience, spectrum sensing data are abbreviated as sensing data in the rest of this paper.

Fortunately, SSDF attack can be suppressed by trust mechanism and many efforts have been made to study various trust schemes [5-9]. They estimate whether an SU is trustworthy or not by its historical behaviors and give low weights to the sensing data from less trustworthy SUs when generating a final decision. Meanwhile, these trust schemes are mostly related on central environments, in which the fusion center (FC) is employed to manage CSS and implement trust schemes by involving complex mathematically analysis and computation. However, these central trust schemes cannot be applied to CRAHNs directly due to two reasons: 1) SUs are generally the mobile devices with limited computation capability, 2) no a central authority can be employed to manage CSS with trust schemes. Although the references [10-12] have been made efforts to trust schemes for CRAHNs, they paid no attention to securing multi-channel selection which is the key features of CRAHNs.

To secure multi-channel selection, a distributed trust evaluation (DTE) scheme is proposed in this paper to defend against SSDF attack and its implementation strategies in CRAHNs are also described. The main contributions of this paper are as following:

- Noting that the sensing behavior of each SU is a binary variable, we can evaluate the trust value of each SU through analyzing such binary variable in the DTE scheme, resulting in less mathematical analysis and computation. Without the central authority, the DTE scheme is implemented at each SU with three modules: Direct Trust Evaluation, Indirect Trust Evaluation and Trust Engine. The former two modules are designed from the perspective of direct experience and neighbor help respectively, and Trust Engine invokes them to perform the evaluation of trust value in a distributed manner. 
- Design a distributed election strategy to dynamically elect a few power SUs from the network to play the role of central authority in CRAHNs. Like a democratic system, power SUs are dynamically replaceable, if they become less active or demonstrate unacceptable behavior.

- A distributed trust implementation protocol is designed to implement the DTE scheme with the help of power SUs. Besides securing multi-channel selection, the other advantages of this protocol are as: 1) avoid the conflict when several source SUs request the scarce available channels, 2) reduce the CSS cost during the routing, that is, only the source SU is asked to find an idle spectrum which can be allowed to continue using the idle spectrum as the routing channel until the destination SU receives data packets, 3) avoid the interference to PUs when they come back during the routing process.

The organization of this paper is as follows: in section2, preliminaries are described. We construct the DTE scheme in section 3 and describe its implementation strategies in section 4 including "power SUs election" and "distributed trust implementation protocol". Simulation analysis is given in section 5. Finally, we conclude the paper in section 6 .

\section{Preliminaries}

\subsection{Cooperative spectrum sensing}

In the selection of multi-channel, CSS is the key function of CRAHNs to find the available channel from several PU spectrums. In CRAHNs, SUs cooperate with each other to achieve a CSS exchange in the self-organizing manner due to the lack of centralized control. As shown in Figure 1, when a source SU (such as $S U_{0}$ ) wants to know the spectrum status of several PUs in a CRAHN, a CSS exchange will be trigged: individual sensing, data reporting and data fusion.

- Individual sensing: Each cooperating SU (cooperators) senses the spectrum status of PUs via the sensing channel individually. The sensing chan- 


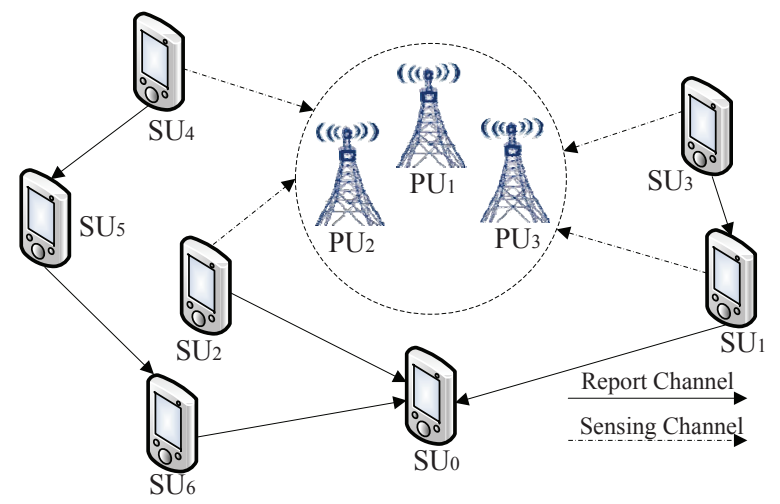

Figure 1: The CSS exchange launched by $S U_{0}$ in a CRAHN.

nel is the selected licensed frequency band where a physical point-to-point link between the PU transmitter and each cooperator for observing the primary spectrum [13].

- Data reporting: All cooperators send their sensing data to $S U_{0}$ via the report channel. The reporting channel is a control channel where a physical point-to-point link between each cooperating SU and the initiator SU is used to send individual sensing information [13]. If both sensing channel and reporting channel are not perfect, an SU observing a weak sensing channel and a strong report channel and another SU with a strong sensing channel and a weak reporting channel, for example, can complement and cooperate with each other to improve the performance of CSS. In Figure 1, $S U_{3}$ and $S U_{4}$, who observe strong PU signals, may suffer from a weak reporting channel. $S U_{1}, S U_{5}$ and $S U_{6}$, who have a strong reporting channel, can serve as relays to assist in forwarding the sensing data from $S U_{3}$ and $S U_{4}$ to $S U_{0}$.

- Data fusion: Without a central authority, $S U_{0}$ combines the received sensing data and to determine the final decision for which PU spectrum is idle. The final decision are usually made according to three typical CSS fusion rules, such as the "AND", "OR" and "Majority" rule [14]. If $P U_{2}$ 
spectrum is idle, it will be used as the available channel for $S U_{0}$.

Typically, individual sensing for primary signal energy detection can be formulated as a binary hypothesis problem as follows [3]:

$$
y(t)=\left\{\begin{array}{lr}
n(t), & H_{0} \\
h(t) \cdot s(t)+n(t), & H_{1}
\end{array}\right.
$$

where $y(t)$ represents the detected signal at each $\mathrm{SU}, s(t)$ is the transmitted PU signal, $h(t)$ is the channel gain of the sensing channel, $n(t)$ is the zero-mean additive white Gaussian noise (AWGN), $H_{0}$ and $H_{1}$ denote the hypothesis of the absence and the presence of the PU signal, respectively. If the estimated energy of the received signal is larger than the decision threshold, the existence of PU would be declared. Otherwise, if the energy of the received signal is smaller than the threshold, there is no PU signal.

After the individual sensing, the individual sensing data of each SU is determined. $d_{i}$ indicates the individual sensing data of $S U_{i}$, which is usually expressed as a binary variable:

$$
d_{i}=\left\{\begin{array}{l}
0, H_{0} \\
1, H_{1}
\end{array}\right.
$$

where " 0 " and " 1 " denote the hypothesis of the absence and the presence of the PU signal, respectively. The spectrum sensing problem therefore can be regarded as a binary. Correspondingly, the final decision $d$ is also binary under the "AND", "OR" and "Majority" rule. In the "AND" rule, FC determines $d=1$ if all individual sensing $d_{i}=1$. The "OR" rule refers to $d=1$ if an individual sensing $d_{i}=1$. The "Majority" rule requires at least a half of SUs to report 1. The "OR" rule works best when the number of SUs is large, whereas the "AND" rule works well when the number of cooperating users is small, and the "Majority" rule can be obtained from the $k$ out of $N$ rule under the condition when $k \geq N / 2$ [13]. 


\subsection{SSDF attack and trust schemes}

Since the individual sensing data is usually regarded as a binary variable,

125 attack by faking sensing data, resulting in a wrong final sensing decision.

Generally speaking, the basic goal of SSDF attackers against CSS in CRAHN$\mathrm{s}$ is to disturb multi-channel selection. Such attackers can be classified according to their attack intention [15].

130

- Always-free: The attackers submit an absent PU signal, although there are PUs using their spectrums. In this case, the source SU makes a wrong decision that the PU spectrums are free, and thus causing interference to $\mathrm{PU}$ in the section of multi-channel. The intention of such attackers is to give interference to PUs.

- Always-busy: The attackers declare that the licensed user is active, although there are no PU signals. In this case, the source SU makes a wrong decision that PUs are present and will not use the spectrum. The intention of such attackers is to gain exclusive access to the target spectrum in the section of multi-channel.

These two types of SSDF attackers are dangerous. Fortunately, they can be detected by trust schemes if malicious SUs always report false sensing data. This is because they will obtain a lower trust value when they always fake sensing data.

Nowadays, trust schemes are increasing influence on many application scenarios, including e-commerce [16], P2P file-sharing [17], ad hoc routing [18], online social networks [19], etc.

Trust schemes also play significant roles in CSS area, such as 1) assisting an initiator SU' accurate decision-making, 2) encouraging honest behaviors, and 3) preventing participating from malicious SUs. Representative trust mechanisms are as follows. In [5], the authors propose a novel trust-aware hybrid spectrum sensing scheme, in which the Beta reputation is used to construct trust scheme. 
Zeng et al propose a reliable CSS scheme with trusted SUs assistance for mitigating SSDF attack in [6]. In [7], the authors consider trust as a competitive factor to punish malicious SUs to access any vacant PU spectrum. In [8], the authors measure the trust of SUs in CSS during the cognition cycle, and incorporate it into the sensing data fusion to reduce the effect of malicious SUs on final decision making. In [9], the SU trust degree is updated according to its behavior in CSS, and used in the sensed resource allocation process. The common property of these existing trust schemes is that the trust value of an $\mathrm{SU}$ is evaluated by his historical sensing behaviors, and low weights are given to the sensing data from less trustworthy SUs when generating a final decision. However, these CSS trust schemes focus on central environments, so they cannot be applied in CRAHNs. Currently, we have found several types of trust schemes for CRAHNs. In [10], the authors propose a novel trust-aware gossipbased CRAHNs that uses push-sum protocol and significantly improves the PU detection performance in the presence of SSDF. In [11], a weighted consensusbased spectrum sensing algorithm with trust assistance is used to protect the spectrum sensing process in a hostile cognitive radio vehicular ad hoc networks. In [12], the authors propose a trust scheme to mitigate SSDF attack and evaluate the performance improvement through extensive Monte Carlo simulations for large-scale cognitive radio ad hoc networks in TV white space. But, how to secure multi-channel selection in CRAHNs is not considered in these trust schemes.

\section{Distributed trust evaluation scheme}

Unlike central cognitive radio networks, CRAHNs lack a central authority to make the cooperative decision. In this case, SUs communicate among themselves and converge to a unified decision on the presence or absence of PUs. Therefore, the CSS exchange of CRAHNs has attracted increasing attention. Specially, there is litter literature investigating the trust scheme for CRAHNs recently. In 


\subsection{Design Philosophy}

Without the central authority, the DTE scheme is obviously implemented at each SU. The architectural view of the DTE scheme is shown in Figure 2, including three modules: Trust Engine, Direct Trust Evaluation and Indirect Trust Evaluation .

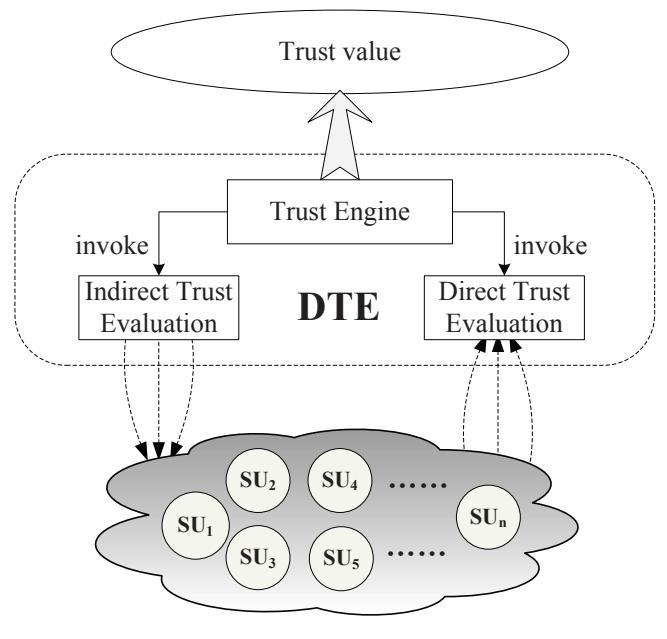

Figure 2: Functional modules in the DTE scheme.

To expose this scheme, we create a scenario to explain how the DTE scheme works at each SU, as shown in Figure 3. For example, when $S U_{6}$ receives all sensing data from cooperators, the Trust Engine module will be triggered by $S U_{6}$ to check whether cooperators are trustworthy.

The working steps of Trust Engine are as follows.

Step 1. Trust Engine judges whether $S U_{6}$ has direct CSS experience (the number of honest or false sensing) with cooperators. For the cooperators with whom $S U_{6}$ has direct CSS experience, Trust Engine invokes the Direct Trust Evaluation module. For the rest of cooperators, go to Step 2.

Step 2. $S U_{6}$ asks his neighbors for help. For the cooperators with whom neighbors have CSS experience, Trust Engine invokes the Indirect Trust Evaluation module. For the rest of cooperators, go to Step 3. 


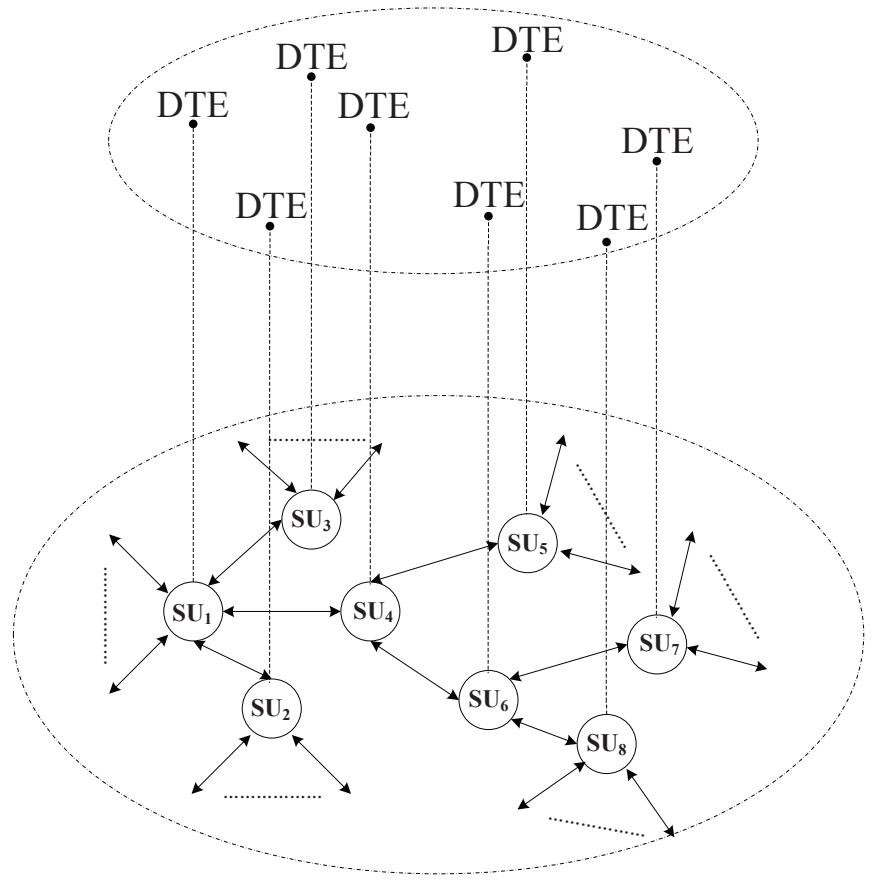

Figure 3: Implementing DTE at each SU.

Step 3. If $S U_{6}$ broadcasts the rest of cooperators with whom both $S U_{6}$ and his neighbors have nothing CSS experience, the network load will be increased seriously. According the features of fusion rules, we can give the initial trust value of newcomers as $\theta$ (the threshold of trust value). Afterwards, the more these newcomers often report honest sensing data for $S U_{6}$, the higher trust value they will get, and vice verse.

Step 4. $S U_{6}$ can choose a CSS fusion rule to fuse all sensing data with trust value. That is, trust value can be used to predict whether cooperators report correct or false sensing data. If a cooperators' trust value is less than $\theta$, he will be marked as a malicious SU since he always report false sensing data which should be filtered out in the data fusion process. Only the cooperators with high trust value (larger than $\theta$ ) can be fused in the data fusion process. 
Step 5. At the end of CSS, Trust Engine checks whether cooperators' sensing data $(s d)$ are consistent with the actual spectrum status (ass) of the target PU. Meanwhile, Trust Engine updates the number of honest sensing (hon) and the number of false sensing ( fal $)$ by Procedure 1 .

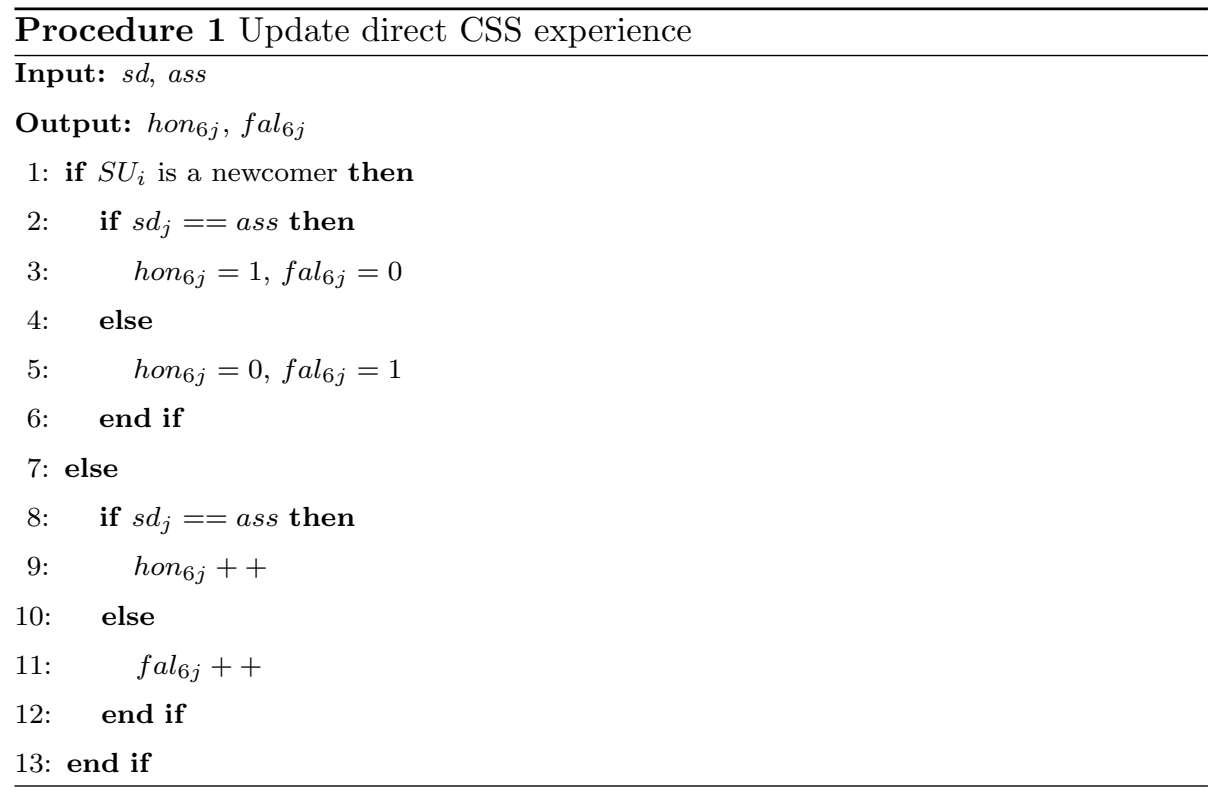

Obviously, the Direct Trust Evaluation and Indirect Trust Evaluation modules are the core of DTE. Then, we present the design methods of the two modules.

\subsection{Direct trust evaluation}

With the direct CSS experience, we can find that each SU may behave two types of sensing results: honest or false. Based on this, we can evaluate trust value by two factors: the number of honest sensing (hon) and the number of false sensing $(f a l)$. Recently, one of the most popular designs to evaluate binary input (i.e., positive or negative) is based on beta function. It first counts the number of honest and false behaviors a user has conducted, and then calculates 225 a value with beta probability density functions (PDF) denoted by $\operatorname{Beta}(\alpha, \beta)$ 
$[19]$.

$$
\operatorname{Beta}(\theta \mid \alpha, \beta)=\frac{\Gamma(\alpha+\beta)}{\Gamma(\alpha) \Gamma(\beta)} \theta^{\alpha-1}(1-\theta)^{\beta-1}
$$

where $\theta$ is the probability of sensing behaviors, $0 \leq \theta \leq 1, \alpha>0, \beta>0$.

Take example for $S U_{i}$ and $S U_{j}, h o n_{i j}$ and $f a l_{i j}$ denote the number of honest sensing and false sensing performed by $S U_{j}$ to $S U_{i}$. The trust value $\left(T_{i j}\right)$ can be evaluated with beta function as: $T_{i j}=\operatorname{Beta}\left(h_{o n} n_{i j}+1, f a l_{i j}+1\right)$. Without any prior observations, $h_{i j}=f a l_{i j}=0$ and hence, $T_{i j}=\operatorname{Beta}(1,1)$.

Consider the condition $\Gamma(x)=(x-1)$ ! when $x$ is an integer [21]. It can be found that the expectation value of the beta function is given by: $E[\operatorname{Beta}(\alpha, \beta)]=\alpha /(\alpha+\beta)$. Therefore, $T_{i j}$ can be further described is as follows:

$$
T_{i j}=\frac{1+\text { hon }_{i j}}{2+\text { hon }_{i j}+\text { fal }_{i j}}
$$

\subsection{Indirect trust evaluation}

Without direct CSS experience, $S U_{i}$ can request help from his neighbors. Let $\mathrm{C}$ denote the set of cooperators with whom $S U_{i}$ 's neighbors have CSS experience and $S U_{j} \in C, H$ denote the set of neighbors who can help $S U_{i}$ and $S U_{k} \in H$. The trust value $T_{i j}$ in the Indirect Trust Evaluation module can be evaluated as:

$$
T_{i j}=\frac{1}{|H|} \sum_{S U_{k} \in H, S U_{j} \in C} r_{k j}
$$

$r_{k j}$ is the trust recommendation on $S U_{j}$ from $S U_{k}$. It is difficult to find evidence to show $r_{k j}$ is honest. But, we can introduce a weight factor $\left(\lambda_{i k}\right)$ to reduce the negative impact of false $r_{k j}$. $\lambda_{i k}$ can be calculated as:

$$
\lambda_{i k}=\left\{\begin{array}{c}
\frac{\left|h r_{i k}-f r_{i k}\right|}{\left|1+h r_{i k}\right|},\left|h r_{i k}-f r_{i k}\right|<\frac{h r_{i k}}{2} \\
\frac{\left|h r_{i k}-f r_{i k}\right|}{\left|1+h r_{i k}+f r_{i k}\right|},\left|h r_{i k}-f r_{i k}\right| \geq \frac{h r_{i k}}{2}
\end{array}\right.
$$

where the parameters $\left(h r_{i k}, f r_{i k}\right)$ are the number of honest or false recommendation performed by $S U_{k}$ to $S U_{i}$. At the end of CSS, $\left(h r_{i k}, f r_{i k}\right)$ are also updated by Procedure 2 . 


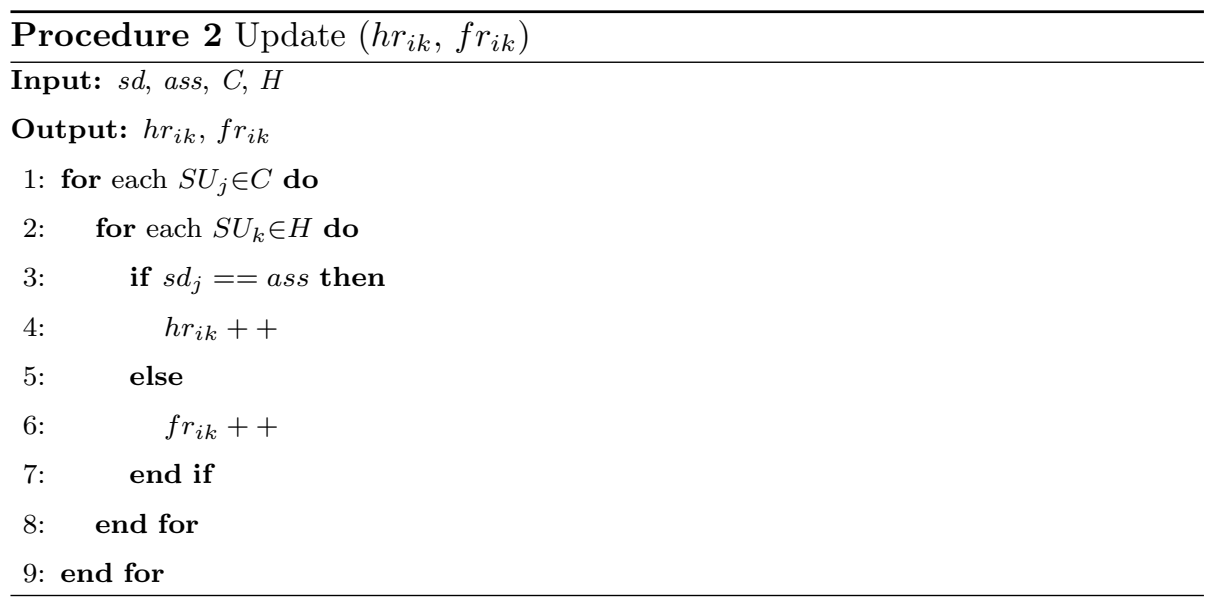

With $\lambda_{i k}$, the more $f r_{i k}$ recommended by $S U_{k}$, the less impact on $T_{i j}$ will be given. Specially, for $f r_{i k} \gg h r_{i k}, \lambda_{i k}$ will become very small. Then, $T_{i j}$ can be further evaluated as:

$$
T_{i j}=\frac{1}{|H|} \sum_{S U_{k} \in H, S U_{j} \in C} \lambda_{i k} * r_{k j}
$$

\section{Implementation strategies}

The effectiveness of supporting trust scheme in CRAHNs depends not only on the factors and metric for evaluating trust value, but also on the implementation of the DTE scheme in a distributed manner. Typical issues in implementing DTE in CRAHNs include "power SUs election" and "distributed trust implementation protocol".

\subsection{Power SUs election}

We have known that it is impossible to employ a central authority to manage CSS among SUs in CRAHNs. In this case, it may cause conflict when more than two SUs request an avaliable channel at the same time. To avoid this conflict, we can dynamically select a few power SUs that are most trustworthy by using a distributed selection strategy. The good trustworthiness of power SUs is accumulated from the running history of the network with DTE. Like 
a democratic system, power SUs are dynamically replaceable, if they become less active or demonstrate unacceptable behavior. Thus, power SUs can be elected dynamically by Procedure 3, based on three strategies: initialization, elimination and replacement.

- Initialization: In a new network, all SUs have nothing on trust value. $n$ SUs are selected randomly to become power SUs at first. Several power SUs are necessary for a network in case there may be the malicious user reports some wrong information for idle channels.

- Elimination: All power SUs must respond to the Query messages from source SUs who request CSS action. However, two unhoped cases should be considered: 1) compared with the majority, the few power SUs may respond the inconsistent $\mathrm{PU}$ information accidentally or intentionally, 2) the few power SUs may reject to respond the QueryHit messages. Generally, the majority vote can be adopted to settle the authentication of PU information among power SUs, since the truth is often in the hands of the majority. For instance, if the PU information reported by a power SU (such as $S U_{h}$ ) is different with the majority vote, it may be identified as fake. According to the majority vote, source SUs can make the judgment whether they can sense a PU spectrum by triggering CSS, but they should discard the fake PU information and send their feedback including the QueryHit message of $S U_{h}$ to all power SUs who will check whether the PU information held in their channels database is consistent with the feedback from source SUs. Meanwhile, they update $S U_{h}$ 's behavior value $\left(f_{h}, q_{h}\right)$ as follows

$$
\text { behavior value } \stackrel{\text { update }}{\rightarrow} \mid \begin{gathered}
f_{h}=f_{h}+1, \text { two unhoped cases ocured in } S U_{h} \\
q_{h}=q_{h}+1, \text { when receive a Query message }
\end{gathered}
$$

Then, the power value of $S U_{h}$ at each power SUs can be calculated as

$$
W_{h}=\frac{q_{h}-f_{h}}{q_{h}+1}
$$


For $W_{h}<\varepsilon$ (threshold of power value), $S U_{h}$ will be kicked out of the group of power SUs. To avoid $q_{h}=0$ in the initialization of network, the denominator of eq.(8) is set to $q_{h}+1$.

- Replacement: To avoid increasing the load of power SUs, we cannot argue that $n-1$ power SUs elect the most trustworthy SU as soon as one power is kicked out. We should wait that $m$ power SUs are kicked out, and then the other $n-m(m \ll n)$ power SUs can elect the $m$ most trustworthy SUs from the network to supplement their group.

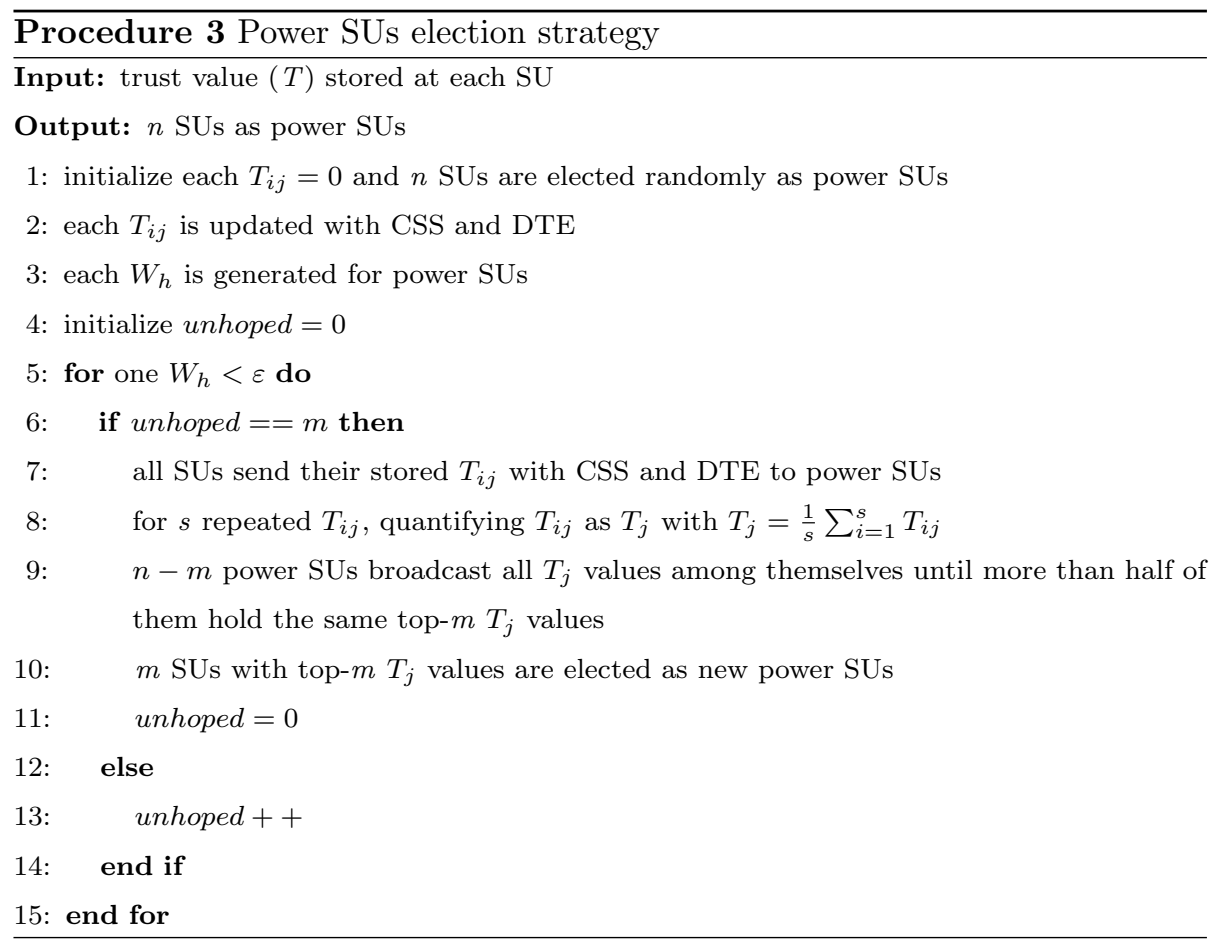

Next, power SUs will play a crucial role in distributed trust implementation protocol for managing CSS and DTE in the process of multi-channel selection.

\subsection{Distributed trust implementation protocol}

To secure multi-channel selection in CRAHNs, only using the DTE scheme to defend against SSDF attack is not enough. Some other important factors 
should be considered: (1) How to avoid the conflict when several SUs request the scarce idle channels? (2) Should each routers trigger CSS and DTE during forwarding data packets? (3) If the PU comes back during forwarding data packets, what can routers do? (4) How to process the available channel when the destination SU received data packets?

Based on the above considerations, a distributed trust implementation protocol with six steps is designed to implement the DTE scheme, as shown in Figure 4. Without the central authority, this protocol is maintained by all SUs' activities. That is, all SUs must listen to the beacon in order to perform the distributed trust implementation protocol with their five types of dynamic activities, including source SU, cooperators, power SUs, routers and destination SU.

- Source SU: An SU who needs a help to find an idle channel sends a Query message to power SUs, and thus triggering the distributed trust implementation protocol.

- Cooperators: The SUs who can sense the spectrum status of a PU share their sensing data with the source SU. Although cooperators are not shown in Figure 4, they actually help the source SU via CSS in Step 3.

- Power SUs: The SUs with high power value are elected dynamically by Procedure 3 to play the role of the central authority together.

- Routers: The SUs are in the transmission path from the source SU to the destination SU.

- Destination SU: The ultimate SU receives the data packets from the source SU.

Step 1. A source $\mathrm{SU}$ (such as $\left.S U_{i}\right)$ sends a Query message $Q[i]=\left(S U_{i}, C S S_{i}, S T_{i}\right)$. $C S S_{i}$ indicates that $S U_{i}$ wants to trigger a CSS action for transferring data packets and requests which can PU spectrum be detected as free channel. $S T_{i}$ is the sending timestamp of $Q[i]$. 


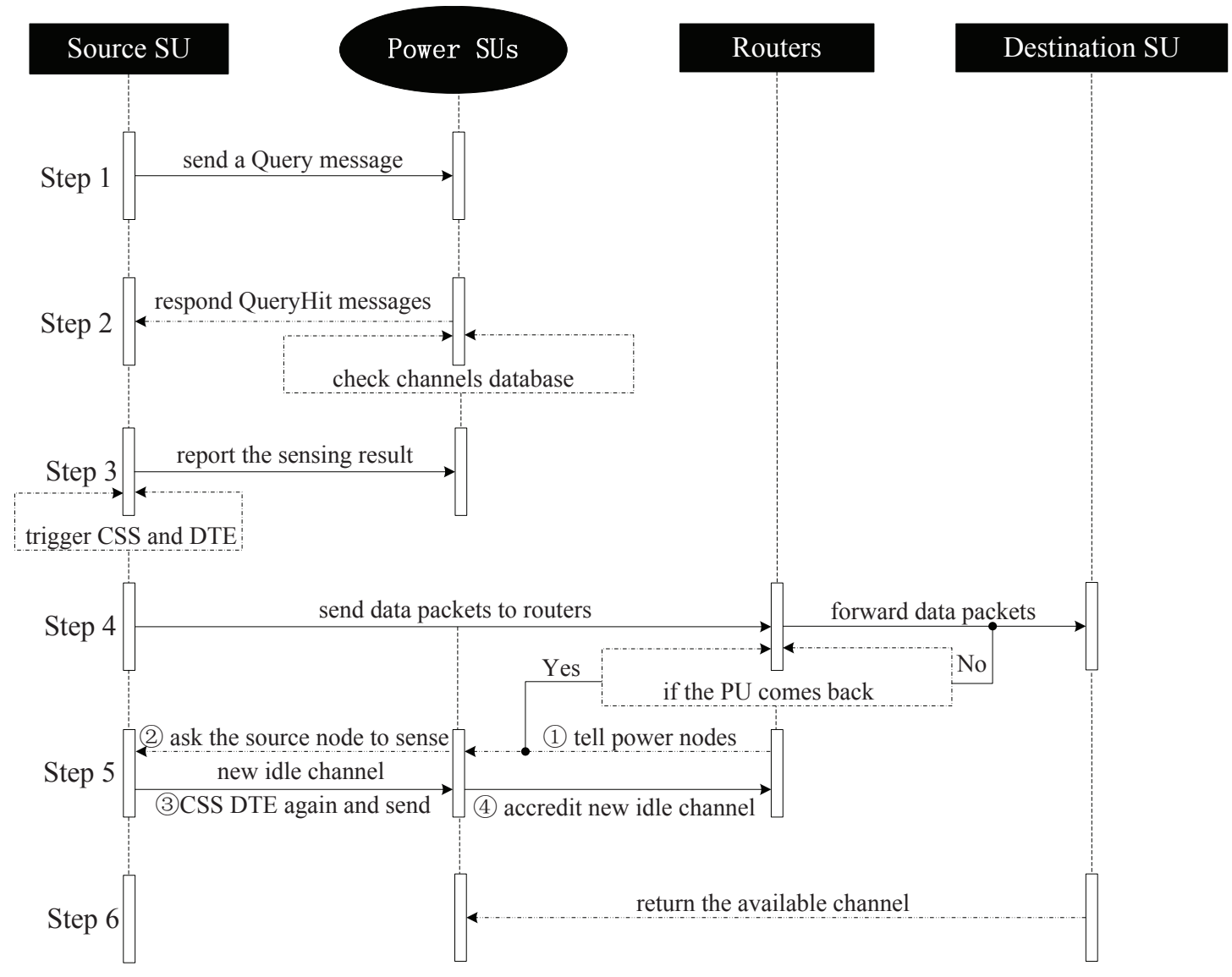

Figure 4: Message flows for the distributed trust implementation protocol.

Step 2. All power SUs must maintain a channels database which contains the ID of PUs and the information of in-use PU spectrums. Upon receiving $Q[i]$, each power $\mathrm{SU}$ checks his channels database and respond a QueryHit message $Q H[k i]=\left(S U_{k}, P U_{t}, S T_{i j}^{k}, R_{i j}\right) . S U_{k}$ is the ID of the $k$-th power SUs. $P U_{t}$ is the ID of the $t$-th PU. $S T_{i j}^{k}$ is the timestamp for $S U_{k}$ 's responding $Q H[i] . \quad R_{i t}=h\left(S U_{i} * P U_{t}\right)$, which means power SUs give the CSS right for $P U_{j}$ to $S U_{i}$. There are two advantages with this equation in $\left.R_{i t}: 1\right)$ all power $\mathrm{SUs}$ respond the same value in $R_{i t}$, so $S U_{i}$ can check whether $Q H[k i]$ is from power $\mathrm{SUs}, 2) h(\cdot)$ is the irreversible hash function 
which can only be held by power SUs, so malicious SUs cannot falsify $R_{i t}$ even though they know the IDs $\left(S U_{i}\right.$ and $\left.P U_{t}\right)$. Specially, to avoid the conflict of the same idle channel, all source SUs and power SUs must follow the three constrained rules in this protocol: 1) all source SUs cannot begin their CSS action until each of them received QueryHit messages $(Q H[k i] \mathrm{s})$, 2 ) power SUs will respond $Q H[k i] \mathrm{s}$ in the order of the sending timestamp, 3) the ID of PUs should be added to $Q H[k i] \mathrm{s}$ in advance when power SUs get the feedback of their idle channels from the destination SUs who have received data packets.

Step 3. If more than half of $Q H[k i]_{\mathrm{s}}$ contain that $P U_{t}$ is ok, $S U_{i}$ will trigger CSS to check whether the $P U_{t}$ spectrum is idle and DTE to enhance the accuracy of cooperative sense result. If not more than half, go back Step 1 and wait new $Q H[k i]$ s. After triggering CSS and DTE, if $P U_{t}$ is idle, $S U_{i}$ reports the sensing result to power SUs and go to Step 4. If not, $S U_{i}$ also reports the sensing result to power SUs and go back Step 1. At the moment of obtaining the sensing result, power SUs mark the $P U_{t}$ spectrum as in-use in their channels database. To save CSS cost in CRAHNs, the marked $P U_{t}$ spectrum can also be used by the routers who are responsible for forwarding the data packets of $S U_{i}$ until $P U_{t}$ comes back or the destination $\mathrm{SU}$ receives the data packets.

Step 4. $S U_{i}$ sends his data packets and sense result to the routing path. If $P U_{t}$ comes back, the routers in the routing path should withdraw from the $P U_{t}$ spectrum and go to Step 5 .

Step 5. Four steps will be performed: (1)The routers tell power SUs. (2)Power SUs send new $Q H[k i] \mathrm{s}$ and ask $S U_{i}$ to sense new idle channel. (3) $S U_{i}$ triggers CSS and DTE again, and then sends the sensing result of new available channel to power SUs. (4)Power SUs accredit the new available channel to the routers to keep up forwarding $S U_{i}$ 's data packets. To avoid collisions with PUs while switching channels, the channel hopping 
sequence must be dynamically adapted to PU activity [22]. Here, the ID of PUs can be denoted as the channel hopping sequence.

Step 6. Upon receiving $S U_{i}$ 's data packets, the destination SU must return the available channel to power SUs as soon as possible.

\section{Simulation analysis}

\subsection{Simulation setup}

Computer simulations are performed to analyze the performance of DTE and its distributed trust implementation protocol (DTIP). The simulation elements are shown in table 1.

Table 1: Description of simulation elements

\begin{tabular}{c|l|c}
\hline \multicolumn{3}{c}{ Table 1: Description of simulation elements } \\
\hline Parameter & \multicolumn{1}{|c}{ Description } & Default \\
\hline$N_{p}$ & Number of PUs in the network & 5 \\
\hline$N_{s}$ & Number of SUs in the network & 60 \\
\hline$n e i$ & Neighbors at each SU & 3 \\
\hline$r h$ & Routing hops & $1 \sim 20$ \\
\hline$T_{\text {threshold }}$ & Threshold of trust value & 0.5 \\
\hline \hline
\end{tabular}

The simulations initiate by cycle-based fashion. At each cycle, all SUs are selected to perform CSS actions with each other randomly. After a few cycles, a trust based network topology is gradually formed by DTE. SUs then use it to perform CSS actions at each cycle, and update the trust value of each SU.

\subsection{Simulation Results}

We have known that the DTE scheme is implemented at each SU. To avoid the overload of CRAHNs, we don't choose the traditional method of global trust recommendation which is very popular in $\mathrm{P} 2 \mathrm{P}$ networks, but introduce the idea of neighbor help to collect recommendation data from neighbors at each SU. Will neighbor help behave well in CRAHNs? The first simulation is performed 


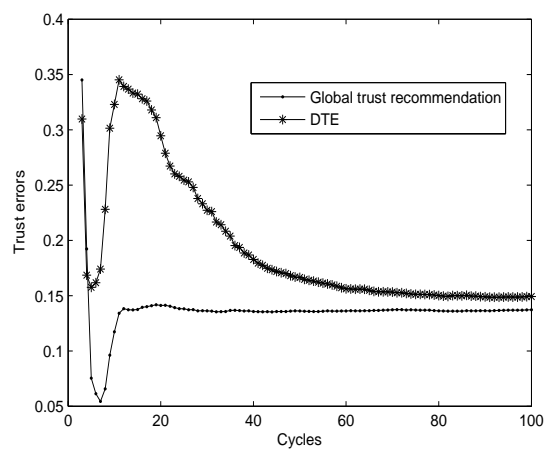

(a)

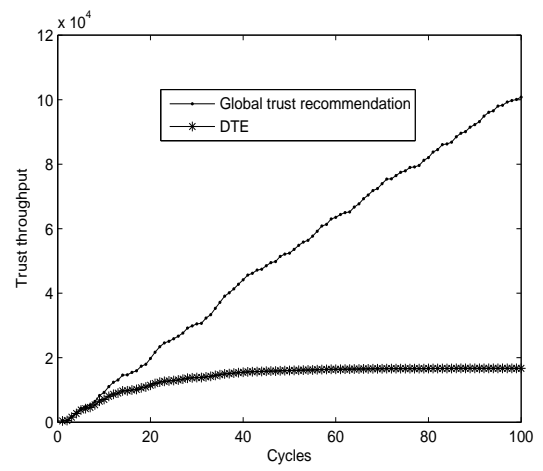

(b)

Figure 5: Global trust recommendation vs. DTE in trust evaluation. (a) trust errors; (b) trust throughput.

to compare DTE with global trust recommendation in terms of trust errors and trust throughput.

The trust errors are specified by:

$$
T E=\frac{1}{T_{s}} \sum_{i=1}^{T_{s}} \sqrt{\frac{1}{T_{i}^{\prime}}\left(T_{i}^{\prime}-T_{i}\right)^{2}}
$$

where $T_{i}^{\prime}$ and $T_{i}$ are the actual and measured trust value of each SU respectively, such as $S U_{i}$.

As shown in Figure 5(a), the trust errors generated by DTE are more than global trust recommendation before 60 cycles. With the increase of cycles, each $\mathrm{SU}$ and his neighbors can get more experiences to evaluate trust value. It can be found that DTE will gradually converge to global trust recommendation. But, we can find in Figure 5(b), the throughput caused by global trust recommendation grows rapidly along with cycles since this method collects recommendation data from the whole network. Due to collecting recommendation data from neighbors, the throughput of DTE is far less than global trust recommendation.

To observe the effectiveness of the DTE scheme in countering SSDF attack, we analyze SSDF attack success ratio against three typical CSS fusion rules, including the "AND", "OR" and "Majority" rule. Two attack patterns are 
considered in this simulation, namely, always-free and always-busy. under the "AND", "OR" and "Majority" rule in some situations. Nevertheless, we find that the damage of SSDF attack to the AND rule is biggest in the always-free attack pattern. The final decision under the "AND" rule is "0" so long as a malicious SU tampers with the sensing data as " 0 ". In the always-busy final decision under the "OR" rule is "1" so long as a malicious SU tampers with the sensing data as " 1 ". Therefore, to make a reliable final decision, the "AND" rule is not a good choice at the always-free attack pattern, and the "OR" rule is not a good choice at the always-busy attack pattern. It can be seen that 395 the damage of attacks against the "Majority" rule amplifies with the number of malicious SUs in the two attack patterns. Malicious SUs can change the final decision when they become the majority. This is because the majority of sensing data are 0 under the "Majority" rule, the final decision is "0". Similarly, the majority of sensing data are "1" under the "Majority" rule, the final decision is "1". Compared with no guard measures, DTE can reduce SSDF attack success ratio for the Majority rule in the two attack patterns. So, the Majority rule is a good choice to make a reliable final decision.

Specially, the DTIP protocol is designed to implement the DTE scheme in CRAHNs. It is not necessary for each router to perform CSS action hopby-hop to search an available channel during forwarding the data packets. In this protocol, only the source SU is asked to search an available channel. The routers can continue to using the available channel until the destination SU receives data packets. Of course, if PUs comes back during the routing process, the routers should withdraw from the PU spectrums and let the source SU find 410 a new available channel using CSS again. $\beta$ is the return probability of PUs during the routing process. Figure 7(a) shows that the throughput generated by DTIP along with cycles is far lower than hop-by-hop CSS. The less is, the lower throughput will be generated by DTIP. During the routing process, Figure 7(b) shows that the throughput generated by DTIP along with hops is also far lower 


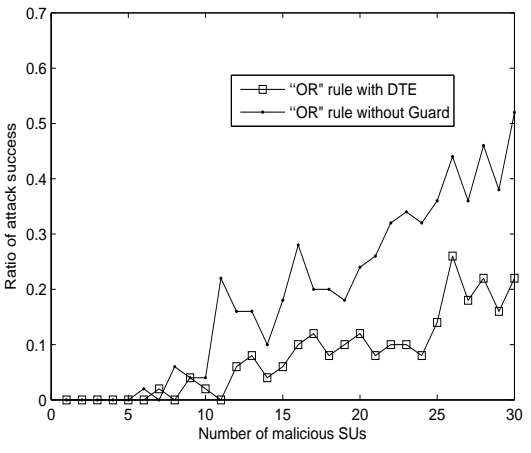

(a) always-free

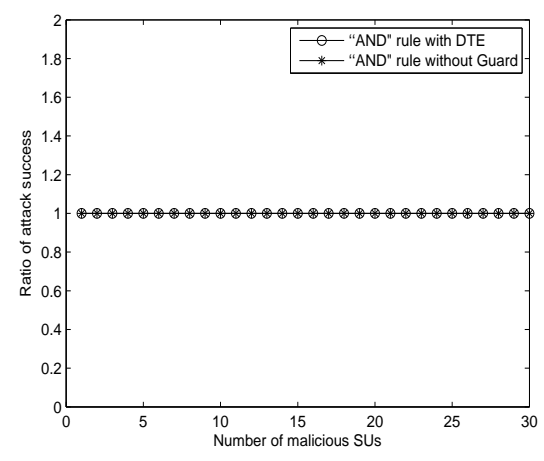

(c) always-free

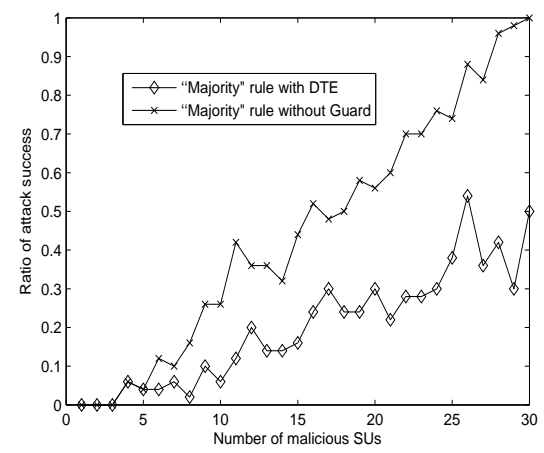

(e) always-free

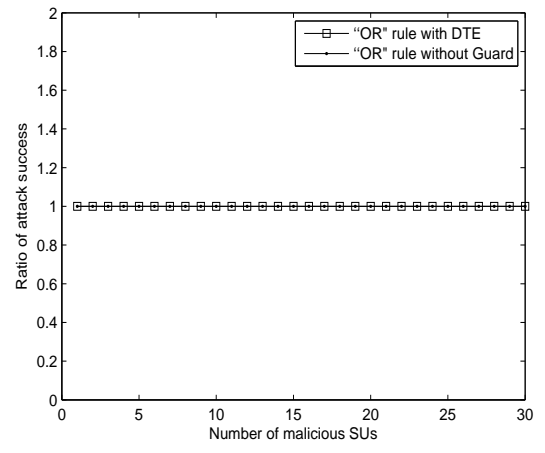

(b) always-busy

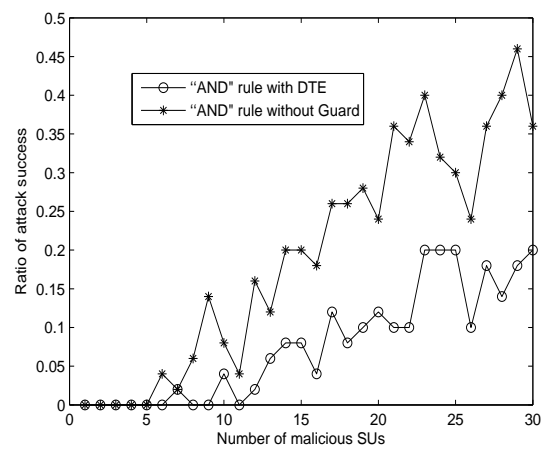

(d) always-busy

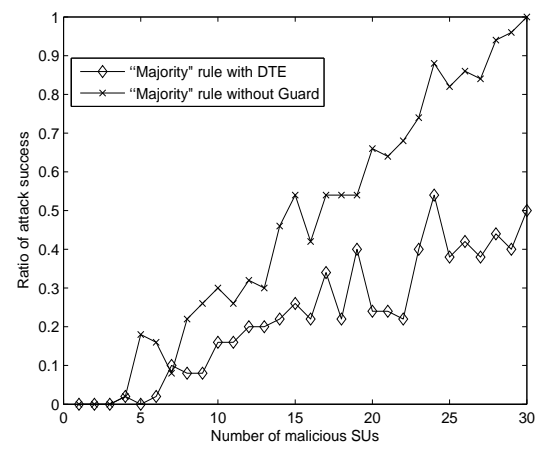

(f) always-busy

Figure 6: SSDH vs. SSDF at attack success rate. 


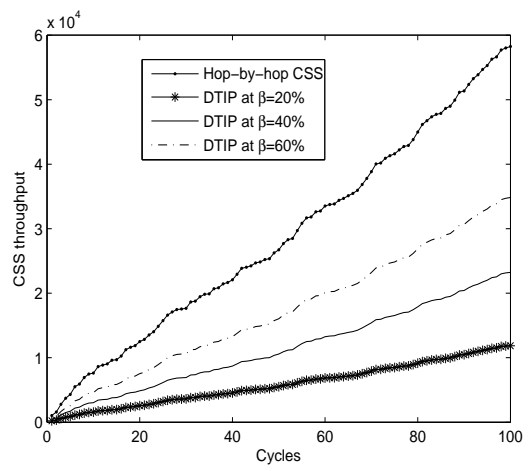

(a)

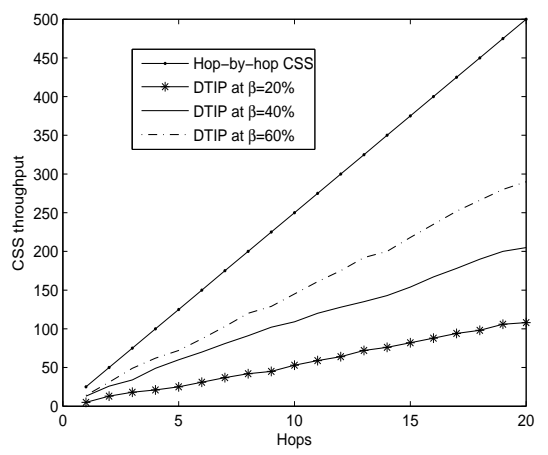

(b)

Figure 7: CSS actions during forwarding data packets. (a) CSS throughput with cycles; (b) CSS throughput with hops.

than hop-by-hop CSS.

\section{Conclusions}

Without the central authority, the multi-channel selection is becoming an attractive target for malicious SUs in CRAHNs. In this paper, we propose a distributed trust evaluation (DTE) scheme from the perspective of direct experience and neighbor help to defend against SSDF attack in CRAHNs. Meanwhile, a distributed trust implementation protocol is designed to implement the DTE scheme in a distributed manner. Power SUs is the managers of this protocol and an election strategy is designed to elect dynamically power SUs. The simulations show that DTE can suppress the SSDF attack success ratio with less trust throughput, and the distributed trust implementation protocol can reduce the throughput of CSS during the routing process.

\section{References}

[1] Federal Communications Commission, 2002. Spectrum policy task force. http://transition.fcc.gov/sptf/files/0801fcc.pdf (accessed 15.08.09). 
[2] Le The Dung, Beongku An, 2015. How do forwarding schemes influence the multi-hop connectivity in cognitive radio ad-hoc networks? In: Proceedings of the seventh international conference on ubiquitous and future networks. pp.805-807.

[3] Akyildiz I.F., Lee W.Y., Chowdhury K. R.,2009. CRAHNs: cognitive radio ad hoc networks. Ad Hoc Netw.7(5): 810-836.

[4] Chen R.L., Park J.M., Hou Y.T., 2008. Toward secure distributed spectrum sensing in cognitive radio networks. IEEE Commun. Magaz. 46(4): 50-55.

[5] Qin T., Yu H., Leung C., 2009. Towards a trust-aware cognitive radio architecture,. ACM SIGMOBILE Mobile Computing and Commun. Review. 13(2): 86-95.

[6] Zeng K., Peng Q.H., Tang Y.X., 2014. Mitigating spectrum sensing data falsification attacks in hard-decision combining cooperative spectrum sensing. Science China. 57(4): 1-9.

[7] Feng J.Y., Lu G.Y., Chang H., 2015. Behave well: how to win a pop vacant band via cooperative spectrum sensing. KSII Trans.on Internet and Inform. Syst. 9(2): 1321-1336.

[8] Pei Q.Q., Yuan B.B., Li L., et al., 2013. A sensing and etiquette reputationbased trust management for centralized cognitive radio networks. Neurocomputing. 101: 129-138.

[9] Chen H, Zhou M, Xie L, et al. Joint spectrum sensing and resource allocation scheme in cognitive radio networks with spectrum sensing data falsification attack. IEEE Transactions on Vehicular Technology, 2016:1-1.

[10] Vosoughi A, Cavallaro J R, Marshall A. A cooperative spectrum sensing scheme for cognitive radio ad hoc networks based on gossip and trust. Signal and Information Processing. IEEE, 2014. 
[11] Wei Z, Yu F R, Boukerche A. Cooperative Spectrum Sensing with Trust Assistance for Cognitive Radio Vehicular Ad hoc Networks. ACM Symposium on Development and Analysis of Intelligent Vehicular Networks and Applications. ACM, 2015:27-33.

[12] Vosoughi A, Cavallaro J R, Marshall A. Trust-aware Consensus-inspired Distributed Cooperative Spectrum Sensing for Cognitive Radio Ad Hoc Networks. IEEE Transactions on Cognitive Communications \& Networking, vol. 2, no. 1, pp. 24-37, March, 2016.

[13] Akyildiz I.F., Lo B. F., Balakrishnan R., 2011. Cooperative spectrum sensing in cognitive radio networks: A survey. Physical Commun. 4(1): 40-62.

[14] Peh E., Liang Y.C., Guan Y.L., et al., 2009. Optimization of cooperative sensing in cognitive radio networks: a sensing-throughput tradeo view. IEEE Trans. on Vehicular Tech. 58(9): 5294-5299.

[15] Rif-Pous H., Blasco M., Garrigues C., 2011. Review of robust cooperative spectrum sensing techniques for cognitive radio networks. Wireless Personal Commun.67(2): 175-198.

[16] Morid M.A., M Shajari., 2012. An enhanced e-commerce trust model for community based centralized systems. Electronic Commerce Research. 12(4): 409-427.

[17] Li X.Y, Zhou F. and Yang X.D. Scalable Feedback Aggregating (SFA) Overlay for Large-Scale P2P Trust Management. IEEE Transactions on Parallel and Distributed Systems. vol. 23, no. 10, pp. 1944-1957, October, 2012.

[18] Boukerche A., Ren Y., Pazzi R., 2009. An adaptive computational trust model for mobile ad hoc networks. In: Proceedings of the 5th International Conference on Wireless Communications and Mobile Computing. pp. 191195. 
[19] Li M, Xiang Y, Zhang B, et al. A trust evaluation scheme for complex links in a social network: a link strength perspective. Applied Intelligence, vol. 44, no.4, pp. 969-987, June 2016.

[20] J $\phi$ sang A., Ismail R., 2002. The beta reputation system. In: Proceeding the 15th Bled Electronic Commence Conference. pp.1-14.

[21] Gamma function. http://en.wikipedia.org/wiki/Gamma_function (accessed 15.10.11).

[22] Misic J., Misic V., 2014. Probabilistic vs. Sequence based Rendezvous in $490 \quad$ Channel-Hopping Cognitive Personal Area Networks. IEEE Trans.on Parallel and Distributed Computing. 25(9): 2418-2427. 
Jingyu Feng received his B.S. degree from Lanzhou University of Technology, China, in 2006. He received his Ph.D. degree from Xidian University, China, in 2011. He is currently a vice professor in Department of Communication Engineering, Xi'an University of Post \& Telecommunication, China. His main research interests include wireless security, trust management and cooperative spectrum sensing.

Guanghua Zhang received his B.S. degree from Heibei Normal University, China, in 2002. He received his M.S. and Ph.D. degree from Xidian University, China, in 2005 and 2014 respectively. $\mathrm{He}$ is currently a vice professor in College of Information Science and Engineering, Hebei University of Science and Technology, China. His main research interests include wireless security, trust management and cooperative spectrum sensing.

Xu Du received his B.S.degree from Xi'an International University, China, in 2015. He is curre ntly a postgraduate Student in Xi'an University of Posts \& Telecommunications. His main researc $\mathrm{h}$ interests include Communication network planning and big data analysis.

Shi Wei received her B.S. and M.S. degree from Xi'an University of Science and Technology, Chi na, in 2003 and 2006 respectively. She is currently a lecturer in Department of Communication Engineering, Xi'an University of Posts \& Telecommunications. Her main research interests includ e Communication network planning and big data analysis. 


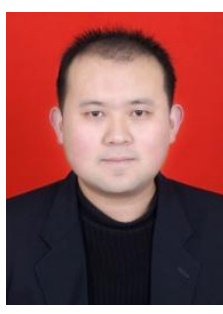

Jingyu Feng

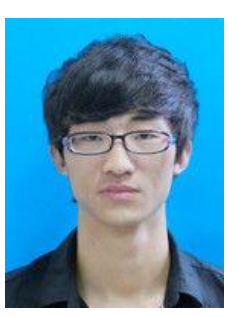

Xu Du

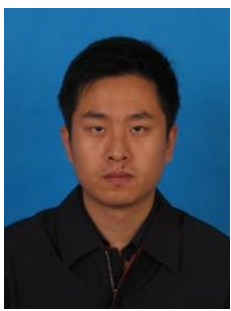

Guanghua Zhang

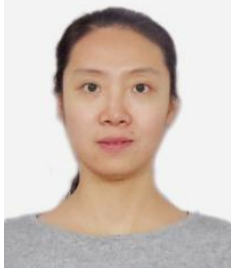

Wei Shi 